\title{
A INFLUÊNCIA DOS FATORES DE RISCO PARA DOENÇAS CARDIOVASCULARES SOBRE A MODULAÇÃO AUTONÔMICA CARDÍACA
}

\author{
THE INFLUENCE OF RISK FACTORS FOR CARDIOVASCULAR DISEASES ON CARDIAC \\ AUTONOMIC MODULATION
}

\section{Pedro Henrique Rodrigues ${ }^{a^{*}}$, Mariana Borges de Oliveira ${ }^{b * *}$, Leonardo Cazelato ${ }^{c^{*}}$, Eduardo Federighi Baisi Chagas ${ }^{\mathrm{d}^{*}}$, Robison José Quitério ${ }^{\mathrm{e}^{*}}$}

\author{
apedrohr.edfisica@gmail.com, bma.boliveira@hotmail.com, cleocazelato@yahoo.com.br, defbchagas@gmail.com, erobisonq@gmail.com \\ *Universidade Estadual Paulista - Rio Claro (SP), Brasil \\ **Universidade Estadual Paulista - Marília (SP), Brasil
}

Data de recebimento do artigo: 18/11/201 Data de aceite do artigo: 04/04/2016

\section{RESUMO}

Introdução: Alterações do sistema nervoso autônomo são fatores de risco independentes para eventos cardiocirculatórios, os quais podem ser estudados pela análise da variabilidade da frequência cardíaca (VFC). Objetivo: Investigar a influência dos fatores de risco para doenças cardiovasculares e dos fármacos vigentes sobre a modulação autonômica da frequência cardíaca de repouso. Métodos: Amostra composta por 23 adultos com pelo menos um fator de risco cardiovascular, com idade entre 50 e 70 anos. Foram realizadas anamneses e avaliações antropométricas. $\mathrm{Na}$ condição de repouso sentado, a frequência cardíaca (FC) e os intervalos R-R foram analisados nos domínios do tempo (FC e RMSSD), da frequência (LF, HF e LF/HF) e pelo plot de Poincaré (SD1, SD2 e SD1/SD2). Resultados: Houve correlaçáo entre presença do sedentarismo e alteraçôes dos índices $\mathrm{LF}(\mathrm{nu})$ e $\mathrm{LF} / \mathrm{HF}(\mathrm{R}=0,479$ e $\mathrm{R}=0,439)$, bem como entre a quantidade de fatores de risco e a $\mathrm{FC}(\mathrm{R}=0,512)$. Conclusão: $\mathrm{O}$ sedentarismo está associado à hiperativaçấo da modulação simpática, e um maior número de fatores de risco para doenças cardiovasculares está associado à elevação da FC de repouso, caracterizando maior estresse sobre o sistema cardiovascular. Já o uso do inibidor da enzima conversora de angiotensina II está relacionado às reduçôes da modulação simpática e ao balanço simpato-vagal, o que é um indicativo favorável para o controle da morbidade.

Palavras-chave: Sistema Nervoso Autônomo; fatores de risco; doenças cardiovasculares.

\section{ABSTRACT}

Introduction: Autonomic nervous system changes are independent risk factors for cardiocirculatory events, which can be studied by analysis of heart rate variability (HRV). Objective: To investigate the influence of risk factors for cardiovascular disease and medications on rest autonomic modulation of heart rate. Methods: A sample of 23 adults with at least one cardiovascular risk factor, aged 50 and 70 years. Case histories and anthropometric evaluations were conducted. In the sitting condition of rest, heart rate (HR) and RR intervals were analyzed in the time domain (HR and RMSSD), frequency (LF, HF and LF/ $\mathrm{HF}$ ), and the Poincaré plot (SD1, SD2 and SD1/SD2 ). Results: There was a correlation between the presence of a sedentary lifestyle and changes in LF (nu) and LF/HF $(R=0.479$ and $R=0.439)$, as well the number of risk factors and the FC $(\mathrm{R}=0.512)$. Conclusion: A sedentary lifestyle is associated with hyperactivation of the sympathetic modulation, and a greater number of risk factors for cardiovascular disease is associated with elevated resting HR, characterizing increased stress on the cardiovascular system. But the Angiotensin II converting enzyme inhibitors is related to reductions in sympathetic modulation and sympathetic-vagal balance, which is a favorable indicator for morbidity's control.

Keywords: Autonomic Nervous System; risk factors; cardiovascular diseases. 


\section{Introdução}

As doenças cardiovasculares são responsáveis por aproximadamente $30 \%$ dos óbitos no Brasil, sendo $50 \%$ desses em plena fase produtiva, entre 30 e 69 anos de idade ${ }^{1}$.

As causas dessas doenças incluem fatores de risco modificáveis biológicos (hipertensão, diabetes e dislipidemias) e outros relacionados ao estilo de vida (obesidade, tabagismo, consumo de álcool e sedentarismo). Já os fatores de risco não modificáveis incluem a idade, gênero, etnia e hereditariedade ${ }^{2}$.

Tem sido constatado que os fatores de risco para doenças cardiovasculares (FRC), como o excesso de gordura corporal, hipertensão arterial, hiperglicemia e hiperinsulinemia, estão associados a alteraçóes na modulação autonômica cardíaca, representando um importante indicador do estado de saúde e fator prognóstico de morbi-mortalidade, independentemente do protocolo de mensuraçáa ${ }^{3}$. Esses motivos, somados à facilidade de aquisição desse recurso, é o que justifica a avaliação clínica da modulação autonômica da frequência cardíaca ${ }^{4}$.

Um dos métodos mais utilizados para investigaçáo do sistema nervoso autônomo (SNA) é o estudo da variabilidade da frequência cardíaca (VFC), que é a análise das oscilaçóes periódicas entre dois batimentos cardíacos consecutivos (iRR) modulados pelo SN simpático e parassimpático. Um alto desvio padrão dos iRR é sinal de boa adaptação, caracterizando um indivíduo saudável e com mecanismos autonômicos eficientes. Por outro lado, um baixo desvio padrão dos iRR é indicador de adaptação anormal e insuficiente do SNA, significando mau funcionamento fisiológico, muitas vezes causado pela presença de morbidades de risco cardiocirculatórias ${ }^{4}$.

As mulheres parecem apresentar melhor controle neurocardíaco que os homens até a menopausa, fase em que ocorrem alterações fisiológicas e de composição corporal, náo sendo mais encontradas diferenças entre os sexos no que se refere à atividade simpato-vagal ${ }^{3}$. $\mathrm{O}$ hipoestrogenismo é fator importante na redução da proteção e aumento do risco cardiovascular porque se relaciona ao aumento da gordura corporal, reduçáo da massa magra e piora no controle glicêmico e do perfil lipídico5.

O estilo de vida atual tem tornado o ser humano cada vez mais propenso aos FRC, situaçóes em que o desequilíbrio autonômico pode ser a via final comum entre uma série de distúrbios e condiçóes para essas doenças, tornando-se um tema importante para estudos e pesquisas.

Dessa forma, este estudo teve como objetivo investigar a influência dos fatores de risco para doenças cardiovasculares e dos fármacos vigentes sobre a modulação autonômica cardíaca de repouso.

\section{Metodologia}

O estudo foi aprovado pelo Comitê de Ética em Pesquisa Envolvendo seres Humanos da Faculdade de Filosofia e Ciências de Marília da Universidade Estadual Paulista, de acordo com a Resolução 466/12 e suas Complementares do Conselho Nacional de Saúde sob parecer de número 1106/2014.

A amostra foi composta de 23 elementos (10 homens e 13 mulheres) com idade entre 50 e 70 anos, que tinham pelo menos um fator de risco para doenças cardiovasculares.

Os fatores considerados para o estudo foram: obesidade global e central, sedentarismo, hipertensão arterial sistêmica, diabetes tipo 2 e dislipidemias com diagnóstico médico, além de vigência de medicamentos para a referida doença. O diagnóstico de síndrome metabólica foi atribuído aos pacientes que apresentaram a presença de três ou mais desses fatores de risco ${ }^{6}$.

Não foram incluídos aqueles pacientes com: diagnóstico de diabetes gestacional; doenças cardíacas, pulmonares e neurológicas; anemia; marca-passo; tabagismo; etilismo; processos infecciosos; alteraçôes eletrocardiográficas; e mulheres não menopausadas (menos de 12 meses sem menstruação).

Os experimentos foram realizados no mesmo período da tarde para padronizar as influências das variaçóes circadianas sobre o organismo. Os voluntários náo ingeriram bebidas alcoólicas e/ou estimulantes e nem realizaram atividades físicas extenuantes 24 horas antes dos testes, além de terem feito uma refeição leve pelo menos 2 horas antes do teste.

A avaliação constou de anamnese; coleta de dados sobre a história pregressa do voluntário e os hábitos de vida. Para avaliar o Nível de Atividade Física Habitual (AFH), foi aplicado o Questionário Internacional de Atividade Física (IPAQ $)^{7}$. Os indivíduos que apresentaram $<150$ minutos por semana de exercício físico moderado a vigoroso nos últimos seis meses foram classificados como sedentários ${ }^{8}$.

Foi realizada a medida de peso corporal e estatura e calculado o índice de massa corpórea (IMC) através da fórmula: massa corporal $(\mathrm{kg}) /$ altura $^{2}(\mathrm{~m})$, classificando os indivíduos como eutrófico (IMC $<24,9 \mathrm{~kg} / \mathrm{m}^{2}$ ), sobrepeso $\left(\right.$ IMC $\left.>25 \mathrm{~kg} / \mathrm{m}^{2}\right)$ e obesidade $\left(\mathrm{IMC}>30 \mathrm{~kg} / \mathrm{m}^{2}\right)^{9}$. No momento da pesagem, os participantes estavam descalços, vestindo apenas roupas leves (camisetas e bermudas).

A obesidade central foi diagnosticada através da medida de circunferência da cintura realizada com o indivíduo em pé, abdômen relaxado e os braços descontraídos ao lado do corpo, utilizando-se uma fita métrica com a precisão de um milímetro posicionada horizontalmente sobre a pele na metade da distância entre as últimas costelas e a crista ilíaca. Considerou-se como risco aumentado os homens e mulheres com circunferência maiores que 94 e $80 \mathrm{~cm}$, respectivamente. 
A FC e os intervalos R-R (iR-R) foram gravados por um sistema digital de telemetria, que consiste de um transmissor posicionado na altura do processo xifóide e um monitor/receptor (Polar RS800CX, Polar Electro Oy, Kempele, Finland). O sistema detecta a despolarizaçáo ventricular, que corresponde à onda $\mathrm{R}$ do eletrocardiograma, com frequência amostral de $500 \mathrm{~Hz}$ e resoluçáo temporal de ms, sendo validado previamente ${ }^{10}$.

$\mathrm{O}$ avaliado foi posicionado sentado e permaneceu em repouso, com o mínimo de movimentos e sem conversar até que as variáveis fisiológicas se estabilizassem. $\mathrm{Na}$ sequência, foram feitos os registros durante 20 minutos com respiração espontânea. Também foram aferidos os valores de pressão arterial pelo método auscultatório segundo as Diretrizes Brasileiras de Hipertensão Arterial².

Os dados foram transmitidos para um computador e filtrados utilizando-se o software Polar ProTrainer (versão 5). Em seguida, foram analisadas somente as séries com mais de $95 \%$ de batimentos sinusais, das quais foram selecionados 256 pontos mais estáveis (Software Kubios HRV, versão 2.0, University of Kuopio, Finland).

No domínio do tempo foram feitos os seguintes cálculos estatísticos: média dos valores instantâneos de FC em batimentos por minuto (bpm); raiz quadrada da média dos quadrados das diferenças entre os intervalos normais sucessivos (RMSSD), expresso em ms. Esse último índice é representativo da modulação parassimpática ${ }^{4,11}$. Foram considerados como normalidade RMSSD $>27 \mathrm{~ms}$ (Task Force, 1996) e para a FC foi $<90$ bpm $^{12}$.

Para a análise no domínio da frequência foi aplicado o método de interpolaçáo splines cúbicas com frequência de $4 \mathrm{~Hz}$ e calculada a densidade de potência espectral do trecho mais estável através da Transformada Rápida de Fourier (FFT), que decompóe o sinal nas seguintes bandas: alta frequência (HF - High Frequency $-0,15$ a $0,4 \mathrm{~Hz})$, correspondente à modulação respiratória e do nervo vago (parassimpático) sobre o coração; baixa frequência (LF - Low Frequency - 0,04 a 0,15Hz), representante da modulação simpática e parassimpática, porém com o predomínio da simpática; e a razão $\mathrm{LF} / \mathrm{HF}$, que representa o balanço simpato-vagal ${ }^{4,11}$. Os dados de LF e HF foram apresentados em valores absolutos $\left(\mathrm{ms}^{2}\right)$ e em unidade normalizada (nu). Os dados normalizados foram calculados dividindo-se a densidade espectral de potência de uma dada banda pela potência total, subtraindo a banda de muito baixa frequência e multiplicando por 100. Valores considerados normais foram: $\mathrm{HF}(\mathrm{nu})>29$; $\mathrm{LF}(\mathrm{nu})<54$; $\mathrm{LF} / \mathrm{HF}<2^{11}$.

A análise não linear foi obtida pelo plot de Poincaré, que é um mapa de pontos em coordenadas cartesianas em que cada ponto é representado, no eixo horizontal $\mathrm{X}$ (abscissa), pelo intervalo RR normal precedente e, no eixo vertical Y (ordenada), pelo intervalo RR seguinte. $O$ desvio padrão dos pontos perpendiculares e ao longo da linha de identidade dá origem aos índices SD1 e SD2, respectivamente. $\mathrm{O}$ índice $\mathrm{SD} 1$ mede o desvio padrão das distâncias dos pontos à diagonal $y=x$, está relacionado à variabilidade em curto prazo, é influenciado pela arritmia sinusal respiratória e representa a atividade parassimpática. O SD2 mede o desvio padrão das distâncias dos pontos à reta $y=-x+R R m$, no qual $R R m$ é a média dos iR-R com variabilidade a longo prazo e reflete a variabilidade global. A relação de ambos (SD1/SD2) mostra a razão entre as variaçóes curta e longa dos $i R-R^{13}$.

Os resultados foram apresentados pela estatística descritiva com média e desvio padrão (DP). As variáveis categóricas estão descritas pela distribuiçấo de frequência relativa. A distribuição de normalidade foi verificada pelo teste de Shapiro-Wilk. Foi utilizado teste de Pearson (paramétrico) e o de Spearman (não paramétrico) para analisar a correlação entre variáveis quantitativas. $\mathrm{O}$ teste de Kendall foi utilizado para correlacionar variáveis qualitativas dicotômicas. Na correlação, foram considerados os valores: 0 a 0,25 correlação fraca; 0,25 a 0,50 correlação razoável; 0,50 a 0,75 correlação moderada; $>0,75$ correlaçáo forte ${ }^{14}$. Foi utilizado o teste $t$ de Student (paramétrico) e o teste de Mann-Whitney (não paramétrico) para comparação de médias. O nível de significância adotado foi de $5 \%(\mathrm{p} \leq 0,05)$. Os dados foram analisados no software SPSS, versão 20.0 para Windows.

\section{Resultados}

A amostra apresentou: idade $=59,8 \pm 5,9$ anos; IMC $=$ $29,7 \pm 5,9 \mathrm{~kg} / \mathrm{m}^{2}$; circunferência de cintura $=100,5 \pm 14,3$ $\mathrm{cm}$; Pressão Arterial Sistólica $=120,6 \pm 12 \mathrm{~mm} / \mathrm{Hg}$; Pressão Arterial Diastólica $=78,7 \pm 8,9 \mathrm{~mm} / \mathrm{Hg}$.

Quanto à distribuição de frequência dos fatores de risco na amostra, a hipertensão arterial sistêmica e a obesidade abdominal (circunferência de cintura) apresentaram as maiores prevalências, ambas com 82,6\%, seguidas pela dislipidemia com $73,9 \%$, diabetes tipo 2 com 30,4\%, obesidade geral (IMC $\geq 30$ ) com $26,1 \%$ e sedentarismo com 17,4\%. Quanto à simultaneidade de fatores de risco, 56,5\% foram considerados com síndrome metabólica (mais de três fatores de risco presentes ${ }^{6}$ ).

Os medicamentos vigentes na amostra foram: antagonista de receptores de angiotensina $(47,8 \%)$; hipocolesterolêmico $(39,1 \%)$; bloqueador plaquetário (39,1\%); betabloqueador (39,1\%); hipoglicemiantes (26,1\%); diuréticos $(21,7 \%)$; inibidor da enzima conversora de angiotensina II $(21,7 \%)$; inibidores de canal de cálcio $(17,4 \%)$ e hormônios da tireoide $(4,3 \%)$.

Os dados da FC e de sua variabilidade apresentados na amostra são: $\mathrm{FC}=67,6 \pm 13,2 \mathrm{bpm}$; $\mathrm{RMSSD}=22,2 \pm 11,9$ $\mathrm{ms} ; \quad \mathrm{HF}=176,1 \pm 139,8 \mathrm{~ms}^{2} ; \quad \mathrm{LF}=214,6 \pm 175,5 \mathrm{~ms}^{2}$; $\mathrm{HF}(\mathrm{nu})=43,5 \pm 18,5 ; \mathrm{LF}(\mathrm{nu})=56,4 \pm 18,5 ; \mathrm{LF} / \mathrm{HF}=1,9 \pm 1,7$; 
$\mathrm{SD} 1=15,7 \pm 8,4 \quad \mathrm{~ms} ; \quad \mathrm{SD} 2=39,1 \pm 21,2 \quad \mathrm{~ms} ; \quad \mathrm{SD} 1 /$ $\mathrm{SD} 2=0,41 \pm 0,16$.

$\mathrm{Na}$ Tabela 1 é apresentado o teste de correlação entre a presença de fatores de risco e a normalidade de FC e da VFC. Houve correlação positiva e significativa somente entre sedentarismo e LFun $(\mathrm{R}=0,479)$, entre sedentarismo e $\mathrm{LF} / \mathrm{HF}(\mathrm{R}=0,439)$.

Tabela 1: Teste de correlação entre a presença de fatores de risco e a normalidade de FC e da VFC.

$\begin{array}{lccccc} & \text { FC } & \text { RMSSD } & \text { LF(un) } & \text { HF(un) } & \text { LF/HF } \\ \text { Obesidade IMC } & 0,064 & 0,190 & 0,224 & -0,073 & 0,172 \\ \text { CC aumentada } & 0,178 & -0,147 & 0,210 & 0,242 & 0,250 \\ \text { Sedentarismo (IPAQ) } & 0,163 & 0,147 & 0,479 * & 0,036 & 0,439 \\ \text { Diabetes Tipo 2 } & 0,024 & 0,112 & 0,313 & -0,120 & 0,255 \\ \text { HAS } & -0,163 & 0,094 & -0,020 & -0,314 & 0,020 \\ \text { Dislipidemia } & 0,230 & 0,226 & 0,172 & 0,073 & 0,026 \\ \text { Síndrome } & 0,079 & 0,088 & 0,137 & -0,176 & 0,038 \\ \text { metabólica } & \end{array}$

Nota: ${ }^{*} p \leq 0,05$; HAS = hipertensão arterial sistêmica; IMC = índice de massa corporal; $\mathrm{CC}=$ circunferência de cintura; IPAQ = Questionário Internacional de Atividade Física. Valores referentes ao teste de Kendall.
É apresentado, na Tabela 2, o teste de correlação entre os fatores de risco, quantidade de fatores de risco, FC e os índices da VFC. Foi observada correlação positiva e significativa entre a FC e quantidade de fatores de risco $(\mathrm{R}=0,512)$, na qual o aumento no número de fatores de risco se relacionou ao aumento dos valores de FC.

$\mathrm{Na}$ Tabela 3 é apresentado o teste de comparação das médias da FC e dos índices da VFC entre os indivíduos que têm e não têm síndrome metabólica. Não foi observada diferença para os valores médios dos índices de VFC entre os pacientes com e sem diagnóstico de síndrome metabólica.

A Tabela 4 apresenta a correlação entre a presença dos medicamentos vigentes e os valores de normalidade FC e os índices da VFC. O inibidor da enzima conversora de angiotensina apresentou correlação negativa e significativa com LFnu $(\mathrm{R}=-0,505)$ e com $\mathrm{LF} / \mathrm{HF}(\mathrm{R}=$ $-0,550)$.

Tabela 2: Teste de correlação entre os fatores de risco, quantidade de fatores de risco, FC e os índices da VFC.

\begin{tabular}{|ccccccccccccc} 
& FC & RMSSD & LFms $^{2}$ & LFnu & HFms $^{2}$ & HFnu & LF/HF & SD1 & SD2 & SD1/SD2 \\
\hline IMC & 0,066 & $-0,235$ & 0,070 & 0,367 & $-0,205$ & $-0,366$ & 0,320 & $-0,234$ & 0,068 & $-0,328$ \\
CC & 0,041 & $-0,232$ & 0,025 & 0,324 & $-0,236$ & $-0,324$ & 0,279 & $-0,231$ & $-0,051$ & $-0,205$ \\
\hline PAS & 0,211 & 0,120 & 0,230 & 0,269 & $-0,064$ & $-0,272$ & 0,321 & 0,122 & 0,122 & $-0,141$ \\
\hline PAD & $-0,065$ & $-0,090$ & 0,197 & 0,336 & $-0,063$ & $-0,337$ & 0,320 & $-0,088$ & 0,134 & $-0,246$ \\
\hline No FR & $0,512^{*}$ & $-0,343$ & $-0,102$ & 0,408 & $-0,398$ & $-0,406$ & 0,387 & $-0,342$ & $-0,291$ & $-0,263$ \\
\hline
\end{tabular}

Nota: ${ }^{*} p \leq 0,05 ; \mathrm{IMC}=$ índice de massa corporal; $\mathrm{CC}=$ circunferência de cintura; PAS = pressão arterial sistólica; $\mathrm{PAD}=$ pressão arterial diastólica; $\mathrm{No}$ FR = quantidade de fatores de risco. Valores referentes ao teste de Pearson e de Spearman.

Tabela 3: Teste de comparação das médias da FC e dos índices da VFC entre os indivíduos que têm e não têm síndrome metabólica.

\begin{tabular}{|c|c|c|c|c|c|c|c|}
\hline \multicolumn{8}{|c|}{ Síndrome metabólica } \\
\hline & \multicolumn{3}{|c|}{$\operatorname{Sim}(n=13)$} & \multicolumn{3}{|c|}{ Náo $(n=10)$} & \multirow[b]{2}{*}{$\mathbf{p}$} \\
\hline & Média & \pm & DP & Média & \pm & DP & \\
\hline FC(bpm) & 70,8 & \pm & 14,3 & 63,5 & \pm & 11,0 & 0,072 \\
\hline RMSSD(ms) & 20,7 & \pm & 13,2 & 24,3 & \pm & 10,5 & 0,499 \\
\hline $\mathrm{LF}\left(m s^{2}\right)$ & 178,5 & \pm & 107,3 & 261,6 & \pm & 235,9 & 0,495 \\
\hline $\mathrm{HF}\left(\mathrm{ms}^{2}\right)$ & 171,9 & \pm & 169,0 & 181,6 & \pm & 98,2 & 0,438 \\
\hline LF(nu) & 58,3 & \pm & 18,4 & 54,0 & \pm & 19,5 & 0,594 \\
\hline $\mathrm{HF}(\mathrm{nu})$ & 41,7 & \pm & 18,4 & 45,9 & \pm & 19,5 & 0,600 \\
\hline $\mathrm{LF} / \mathrm{HF}$ & 2,1 & \pm & 1,8 & 1,8 & \pm & 1,7 & 0,577 \\
\hline $\mathrm{SD} 1(\mathrm{~ms})$ & 14,7 & \pm & 9,3 & 17,2 & \pm & 7,4 & 0,500 \\
\hline $\mathrm{SD} 2(\mathrm{~ms})$ & 34,8 & \pm & 11,6 & 44,8 & \pm & 29,5 & 0,203 \\
\hline SD1/SD2 & 0,396 & \pm & 0,158 & 0,442 & \pm & 0,179 & 0,756 \\
\hline
\end{tabular}

Tabela 4: Correlação entre a presença dos medicamentos vigentes e os valores de normalidade FC e os índices da VFC.

\begin{tabular}{cccccc} 
& FC & RMSSD & LF(un) & HF(un) & LF/HF \\
Antidiabético & 0,064 & $-0,018$ & 0,224 & $-0,073$ & 0,172 \\
Hipocolesterolêmico & $-0,046$ & $-0,024$ & $-0,054$ & $-0,207$ & 0,054 \\
Betabloqueador & $-0,311$ & $-0,024$ & $-0,054$ & 0,009 & 0,054 \\
\hline Inibidor da enzima conversora de angiotensina & $-0,204$ & 0,058 & $-0,505^{*}$ & $-0,278$ & $-0,550^{*}$ \\
\hline Antagonista de receptores de angiotensina II & 0,146 & 0,032 & 0,303 & $-0,083$ & 0,394 \\
Diuréticos & $-0,204$ & $-0,164$ & 0,128 & $-0,278$ & 0,083 \\
\hline Inibidor de Canal Cálcio & $-0,178$ & 0,147 & 0,020 & $-0,242$ & $-0,020$ \\
Bloqueador Plaquetário & $-0,046$ & $-0,024$ & $-0,233$ & $-0,207$ & $-0,302$ \\
Hormônios da Tireoide & $-0,083$ & $-0,156$ & $-0,204$ & $-0,112$ & $-0,223$ \\
\hline
\end{tabular}

Nota: ${ }^{\mathrm{p}} \leq 0,05$. Valores referentes ao teste de Kendall. 


\section{Discussão}

Grande parte dos pacientes incluídos no estudo apresentou diagnóstico de obesidade ou pelo IMC $\left(29,7 \pm 5,9 \mathrm{~kg} / \mathrm{m}^{2}\right)$ ou pela circunferência de cintura $(100,5 \pm 14,3 \mathrm{~cm})$. A circunferência de cintura aumentada tem sido associada à elevação dos triglicérides ${ }^{15}$, usualmente acompanhada por diminuição na concentração do colesterol de alta densidade (HDL-c), e oferece maior risco em relação à obesidade periférica, pois aumenta a produção de glicose, secreção de insulina e retenção de sódio ${ }^{16}$.

O IMC está inversamente associado à atividade parassimpática ${ }^{17}$; assim, a frequência cardíaca (FC) em repouso e a VFC se apresentam relativamente maior e menor, respectivamente, em indivíduos obesos em comparação aos não obesos ${ }^{18}$.

Quanto à simultaneidade de fatores de risco, $56,53 \%$ da amostra apresentaram mais de três fatores de risco presentes, caracterizando o diagnóstico de síndrome metabólica $; 30,43 \%$ apresentaram dois fatores; e 13,04\% apresentaram apenas um fator de risco. A hipertensão arterial sistêmica e a circunferência de cintura aumentada estiveram presentes em $82,6 \%$; a dislipidemia em $73,9 \%$; o diabetes tipo 2 , em $30,4 \%$; a obesidade pelo IMC, em 26,1\%; e o sedentarismo, em 17,4\%.

Ferreira et al. ${ }^{19}$ encontraram a prevalência de $80,4 \%$ da amostra com hipertensão arterial; 83,3\%, com obesidade central (CC); 59,8\%, de sedentários; 32,2\%, com obesidade total (IMC); 23,4\%, com dislipidemias; $19,1 \%$, com diabetes, tendo a simultaneidade de dois ou mais fatores de risco ocorrido em $87,3 \%$ da amostra. Petersen et al. ${ }^{20}$ encontraram prevalência de $83,8 \%$ de hipertensos; $67 \%$ de sedentários; $85,6 \%$ com excesso de peso (IMC); e 92,1\% com obesidade visceral (CC), sendo a combinação de fatores de risco identificada em $85,0 \%$ da amostra, e, portanto, com diagnóstico de síndrome metabólica.

Os resultados observados no estudo confirmam a alta prevalência de fatores de risco cardiovasculares na população com idade entre 50 a 70 anos. Embora as prevalências observadas tenham sido influenciadas pelo fato do estudo ter sido realizado em ambulatório clínico e o critério de inclusão tenha sido a presença de ao menos um fator de risco, o crescente aumento da prevalência de fatores risco na populaçáo acima de 50 anos tem representado um grande impacto nos serviços de saúde pública5.

Ao testar a influência dos fatores de risco sobre os valores de FC e VFC, foi observada correlaçáo entre a presença do sedentarismo e alterações na normalidade dos índices $\mathrm{LF}(\mathrm{nu})$ e $\mathrm{LF} / \mathrm{HF}(\mathrm{R}=0,479$ e $\mathrm{R}=0,439)$, apontando influência da inatividade física sobre a hiperativação da modulação simpática ${ }^{21}$.
Analisando a simultaneidade de fatores de risco, foi verificada uma correlaçáo entre a quantidade de fatores e a $\mathrm{FC}(\mathrm{R}=0,512)$, o que aponta maior sobrecarga sobre o aparelho circulatório, provavelmente influenciado pela hiperativação da modulação simpática ${ }^{21}$. Ao compararmos a média dos valores de FC e índices da VFC entre os indivíduos com e sem síndrome metabólica (mais de três fatores de risco $)^{6}$, não foi detectada diferença estatística; porém, o grupo com SM apresentou resultados menos favoráveis em relação ao grupo sem $S M$, o que deve ser considerado na prática clínica. Além disso, o tamanho reduzido da amostra pode ter limitado o poder do estudo em detectar diferenças estatisticamente significantes.

Tem-se observado que os diabéticos podem apresentar neuropatia autonômica devido à degeneração neurológica que afeta pequenas fibras do sistema nervoso periférico e sistema nervoso central, trazendo como consequência a reduçáo da VFC. Singh, Larson e O'donnell ${ }^{22}$ sugerem que esses indivíduos apresentam redução do componente parassimpático devido à exposição à glicemia elevada.

Apesar dos poucos estudos, tem sido verificado que alteraçóes na VFC estão associadas a níveis elevados de colesterol total e lipoproteína de baixa densidade (LDL), visto que a dislipidemia é fator de risco para disfunção endotelial e doença coronariana ${ }^{23}$.

A hipertensão arterial sistêmica e a obesidade central são os fatores de maior prevalência no presente estudo, assim como nos achados de Ferreira et al. ${ }^{19}$ e Petersen et al. ${ }^{20}$. A alta prevalência desses dois fatores aponta diretamente para a relação entre o aumento da circunferência de cintura e a presença de HAS, o que explica a grande utilização de fármacos para controle dessa morbidade na amostra. Em um estudo populacional de Ferreira, Barreto e Giatti ${ }^{24}$, que pesquisou o uso de medicamentos em pessoas com hipertensão referida, $83 \%$ da amostra fazia uso contínuo de medicamentos para controle da morbidade. Motter, Olinto e Paniz ${ }^{25}$ apontaram em seu estudo com 678 hipertensos que o principal fármaco utilizado é o inibidor da enzima conversora de angiotensina II $(58,1 \%$ da amostra).

No presente estudo, ao testar a influência do uso dos medicamentos vigentes sobre as alteraçóes na FC e VFC, foi encontrada correlação do inibidor da enzima conversora de angiotensina II com LF(nu) e LF/HF $(\mathrm{R}=-0,505$ e $\mathrm{R}=-0,550)$, apontando atuação direta desse fármaco sobre o sistema simpático e, consequentemente, sobre o balanço simpato-vagal para controle da patologia.

Vale destacar que todos os pacientes diagnosticados com síndrome metabólica tem HAS, sendo que 61,5\% deles faz uso do medicamento da enzima conversora de angiotensina II, o que pode ter influenciado os 
resultados dos índices de VFC quando comparados entre os grupos com e sem síndrome metabólica.

Assim, o uso de medicamentos ou combinaçóes farmacológicas tem objetivo de melhorar os sintomas e reduzir hospitalizaçóes, apresentando prognóstico cardiovascular mais satisfatório e reduzindo o risco de mor$\mathrm{te}^{26}$. Os medicamentos ajudam no controle e recuperação autonômica principalmente pela reduçáo reflexa da atividade simpática ${ }^{27}$.

A não homogeneidade na distribuição dos fatores de risco e os medicamentos vigentes podem influenciar nos valores de $\mathrm{FC}$ e da modulação autonômica ${ }^{28}$; porém, devido às interaçóes de causa e efeito entre os fatores de risco para doenças cardiovasculares, eles geralmente são concomitantes ${ }^{19,20}$, dificultando a formação de um grupo com apenas um fator de risco e tempo de diagnóstico da doença semelhantes, que utiliza apenas um tipo de medicamento e com a mesma dosagem. Entretanto, essa é a realidade clínica, e compreender a influência desses fatores sobre a modulação autonômica é de extrema importância para estratégias de atenção à saúde.

É comprovada a eficácia dos exercícios físicos regulares na prevenção de FRC, sendo que indivíduos ativos revelam níveis mais elevados de aptidáo associados a níveis relativamente maiores de VFC, se comparados com indivíduos sedentários. Dessa forma, sugere-se a prática de exercícios físicos de forma habitual como uma benéfica forma de preservação da eficiência do sistema nervoso autonômico durante o envelhecimento. Indivíduos ativos apresentam menor risco para eventos cardiovasculares, além de melhor qualidade de vida, incluindo variáveis físicas e psicológicas ${ }^{29}$.

\section{Conclusão}

A partir dos resultados do estudo, conclui-se que o sedentarismo está associado à hiperativação da modulação simpática, e um maior número de fatores de risco para doenças cardiovasculares está associado à elevação da FC de repouso, caracterizando maior estresse sobre o sistema cardiovascular. Já o uso do inibidor da enzima conversora de angiotensina II está relacionado às reduçôes da modulação simpática e ao balanço simpato-vagal, o que é um indicativo favorável para o controle da morbidade.

\section{Referências}

1. Pereira JC, Barreto SM, Passos VMA. Perfil de risco cardiovascular e autoavaliação da saúde no Brasil: estudo de base populacional. Rev Panam Salud Publica. 2009;25(6):491-8.
2. Sociedade Brasileira de Cardiologia/Sociedade Brasileira de Hipertensão/Sociedade Brasileira de Nefrologia. VI Diretrizes Brasileiras de Hipertensão. Arq Bras Cardiol. 2010;95(1 Supl 1):1-51.

3. Kimura T, Matsumoto T, Akiyoshi M, Owa Y, Miyasaka N, Aso T, et al. Body fat and blood lipids in postmenopausal women are related to reting autonomic nervous system activity. Eur J Appl Physiol. 2006;97(5):542-7.

4. Vanderlei LCM, Pastre CM, Hoshi RA, Carvalho TD, Godoy MF. Noçóes básicas de variabilidade da frequência cardíaca e sua aplicabilidade clínica. Rev Bras Cir Cardiovasc. [Internet]. 2009[citado em 2015 out. 12];24(2):205-217. Disponível em: http://dx.doi. org/10.1590/S0102-76382009000200018

5. Chagas EFB, Bonfim MR, Brondino NCM, Monteiro HL. Exercício físico e fatores de risco cardiovasculares em mulheres obesas na pós-menopausa. Rev Bras Med Esporte. 2015;21(1):65-9.

6. Sociedade Brasileira de Cardiologia. I Diretriz Brasileira de Diagnóstico e Tratamento da Síndrome Metabólica. Arq Bras Cardiol. 2005;84 Supl I.

7. Benedetti TRB, Mazo GZ, Barros MVG. Aplicação do Questionário Internacional de Atividade Física para avaliação do nível de atividades físicas de mulheres idosas: validade concorrente e reprodutibilidade teste-reteste. Rev Bras Ci e Mov. 2004;12(1):25-34.

8. Colpani V, Spritzer PM, Lodi NP, Dorigo GG, Miranda IAS, Hahn LB, et al. Atividade física de mulheres no climatério: comparação entre auto-relato e pedômetro. Rev Saúde Pública. 2014;48(2):258-65.

9. World Health Organization. Obesity: preventing and managing the global epidemic. Report of a World Health Organization Consultation. Geneva: World Health Organization; 2000. WHO Obesity Technical Report Series, n. 284.

10. Gamelin FX, Berthoin S, Bosquet L. Validity of Polar S810 Heart Rate Monitor to Measure R-R Intervlas at Rest. Med Sci Sports Exerc. 2006;38(5):887-93.

11. Task Force of The European Society of Cardiology of the North American Society of Pacing Electrophysiology. Heart Rate Variability Standards of Measurement, Physiological Interpretation, and Clinical Use. Circulation. 1996;93(5):1043-65.

12. Yang XS, Beck GJ, Wilkoff BL. Redefining Normal Sinus Heart Rate. J Am Coll Cardiol. 1995;25(2 Suppl 1):193.

13. Brennan M, Palaniswami M, Kamen P. Do existing measures of Poincare plot geometry reflect nonlinear features of heart rate variability. IEEE Trans Biomed Eng. 2001;48(11):1342-7.

14. Dawson B, Trapp RG. Bioestatística básica e clínica. 3. ed. Rio de Janeiro: McGraw-Hill; 2001.

15. Bard JM, Charles MA, Juhan-vague I, Vague P, Andre P, Safar M, et al. Accumulation of triglyceride-rich lipoprotein insubjects with abdominal obesity: the biguanides and the prevention of the risk of obesity (BIGPRO) 1 study. 
Arterioscler Thromb Vasc Biol. 2001;21:407-14.

16. Gomes F, Telo DF, Souza HP, Nicolau JC, Halpern A, Serrano Jr CV. Obesidade e doença arterial coronariana: papel da inflamação vascular. Arq Bras Cardiol. [Internet]. 2010[citado em: 2015 out. 12];94(2):273-9. Disponível em: http://www.arquivosonline.com.br/pesquisartigos/ PDFS/2010/9402/9402021.PDF

17. Santos D, Sichieri R. Índice de massa corporal e indicadores antropométricos de adiposidade em idosos. Rev Saúde Pública [Internet]. 2005[citado em: 2015 out. 12];39(2):163-8. Disponível em: http://www.scielo.br/pdf/ $\mathrm{rsp} / \mathrm{v} 39 \mathrm{n} 2 / 24037 . \mathrm{pdf}$

18. Paschoal MA, Fontana CC. Método do limiar de variabilidade da frequência cardíaca aplicado em pré-adolescentes obesos e não obesos. Arq Bras Cardiol. 2011;96(6):450-6

19. Ferreira CCC, Peixoto MRG, Barbosa MA, Silveira EA. Prevalência de fatores de risco cardiovascular em idosos usuários do sistema único de saúde de Goiânia. Arq Bras Cardiol. 2010;95(5):621-8

20. Petersen LC, Chinazzo H, Saldanha C, Basso M, Garcia P, Bartholomay E, et al. Fatores de risco cardiovasculares e comorbidades em ambulatórios de cardiologia da região metropolitana de Porto Alegre, RS. Rev AMRIGS. 2011;55(3):217-23.

21. Thayer JF, Yamamoto SS, Brosschot JOSF. The relationship of autonomic imbalance, heart rate variability and cardiovascular disease risk factors. Int J Cardiol Heart Vasc. 2010:141(2):122-31.

22. Singh JP, Larson MG, O'donnell CJ, Wilson PF, Tsuji H, Lloyd-Jones DM, et al. Association of hyperglycemia with reduced heart rate variability (The Framingham Heart Study). Am J Cardiol. 2000;86(3):309-12.

23. Christensen JH, Toft E, Christensen MS, Schmidt EB. Heart rate variability and plasma lipids in men with and without ischaemic heart disease. Atherosclerosis. 1999;145:181-6.

24. Ferreira RA, Barreto SM, Giatti L. Hipertensão arterial referida e utilização de medicamentos de uso contínuo no Brasil: um estudo de base populacional. Cad Saúde Pública. 2014;30(4):815-26.

25. Motter FR, Olinto MTA, Paniz VMV. Conhecimento sobre a farmacoterapia por portadores de Hipertensão Arterial Sistêmica. Ciênc Saúde Coletiva. 2013;18(8):2263-74.

26. New York Heart Association. The Criteria Committee of the New York Heart Association. Nomenclature and criteria for diagnosis of diseases of the heart and great vessels. 9. ed. Boston: Little Brown \& Co; 1994.

27. Menezes Júnior AS, Moreira HG, Daher MT. Análise da variabilidade da frequência cardíaca em pacientes hipertensos, antes e depois do tratamento com inibidores da enzima conversora da angiotensina II. Arq Bras Cardiol. 2004;83(2):165-8.

28. Tibana RA, Boullosa DA, Leicht AS, Prestes J. Women with metabolic syndrome present different autonomic modulation and blood pressure response to an acute resistance exercise session compared with women without metabolic syndrome. Clin Physiol Funct Imaging. 2013;33(5):364-72.

29. Meersman RE. Heart rate variability and aerobic fitness. Am Heart J. 1993;125:726-31.

\section{Como citar este artigo:}

Rodrigues PH, Oliveira MB, Cazelato L, Chagas EFB, Quitério RJ.A influência dos fatores de risco para doenças cardiovasculares sobre a modulação autonômica cardíaca. Rev. Aten. Saúde. 2016;14(49):34-40. 